\title{
Initial presentation of lung cancer in the emergency department: a descriptive analysis
}

\author{
Aamir Suhail MD, Candice E. Crocker PhD, Bijon Das MD, Jennifer I. Payne PhD, Daria Manos MD
}

Abstract

Background: Guidelines aimed at improving care for lung cancer, the leading cause of cancer-related death in Canada and worldwide, require accurate knowledge of the diagnostic setting or pathway. We sought to determine how often lung cancer is initially diagnosed through the emergency department.

Methods: We performed a descriptive study that included all cases of primary lung cancer diagnosed in residents of Nova Scotia in 2014. Cancer registry data included diagnostic data and date of death to Aug. 31, 2016. We reviewed linked hospital records, including laboratory and imaging results, to identify the first positive diagnostic study and the route of presentation (emergency department v. other). We evaluated time from diagnosis to death as a function of presentation route using Kaplan-Meier curves and Cox regression (hazard rate ratios [HRRs]).

Results: Sufficient data were available for 946 of 951 cases identified, of which 336 (35.5\%) were diagnosed through the emergency department. Cases diagnosed via the emergency department were more likely to be at an advanced stage (stage IV, $59.5 \%$. $43.4 \%$ ), with patients experiencing shorter survival (1-yr survival, $28.4 \%$ v. $49.5 \%$ ), including stage-specific survival. Mortality for cases diagnosed in the emergency department was 54\% higher than for the non-emergency department group after adjusting for age and stage (HRR 1.54, 95\% confidence interval 1.32-1.81). Few patients $(7.1 \%, n=24)$ who presented to the emergency department reported having no family physician.

Interpretation: The emergency department is a common route of presentation for lung cancer and is associated with advanced stage at diagnosis and reduced survival time. Strategies are needed to encourage pre-emergent diagnosis and to ensure that emergency providers are supported in the initial care of patients with lung cancer.

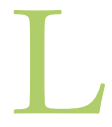

ung cancer remains the leading cause of cancerrelated death worldwide. ${ }^{1}$ Current Canadian estimates include 28600 new cases of lung cancer each year, accounting for $14 \%$ of all new cancer cases and $26 \%$ of all cancer-related deaths. ${ }^{2}$ The high mortality of lung cancer suggests a propensity for patients to present at an advanced stage of disease. In Canada, $68.8 \%$ of lung cancer is diagnosed at an advanced stage (III or IV), ${ }^{2}$ with $56.4 \%$ of stage III and $83.2 \%$ of stage IV patients dying within 1 year of diagnosis. ${ }^{3}$ Late presentation may be caused, in part, by the non-specific nature of early respiratory symptoms in this population. ${ }^{4,5}$ Most patients (67\%) with symptomatic lung cancer present with general respiratory problems, less than one-third of whom report hemoptysis (a clinical sign that prompts more rapid evaluation). ${ }^{4}$ Delays are further exacerbated by the lack of perceived importance of symptoms and stigma associated with smoking-related diseases. ${ }^{6-9}$

Early detection of lung cancer and guidelines for diagnosis are an important public health issue aimed at improving care, improving quality of life, and reducing patient mortality. Consensus guidelines often assume that the evaluation of lung cancer begins in primary care, usually through a family phys- ician who requests diagnostic imaging. ${ }^{10,11}$ The natural history of lung cancer and its propensity to present at a late stage suggests that diagnosis may also occur in an emergent environment. ${ }^{12,13}$ This potentially under-recognized alternative route to diagnosis has implications for both patients and care providers. The emergency department is not always well equipped to deliver a lung cancer diagnosis in terms of starting work-up, ensuring follow-up and providing patient support for immediate questions regarding the diagnosis. These gaps are of particular importance for the growing number of patients who do not have a primary care provider. ${ }^{14}$

We sought to determine the number of lung cancer cases in Nova Scotia that are initially diagnosed through a visit to an emergency department. As a secondary objective, we

Competing interests: None declared.

This article has been peer reviewed.

Correspondence to: Daria Manos, daria.manos@nshealth.ca

CMAJ Open 2019. DOI:10.9778/cmajo.20180061 
compared diagnosis groups (emergency department v. nonemergency department) in terms of patient demographics, diagnostic information and survival.

\section{Methods}

\section{Study design and case identification}

We performed a descriptive study of the route to diagnosis of all cases of primary lung cancer diagnosed in residents of Nova Scotia in 2014. Eligible cases were identified through the Nova Scotia Cancer Registry, which is maintained under the authority of the Nova Scotia Department of Health and Wellness. This population-based registry records information on all new cases of cancer diagnosed in the province and follows Canadian Council of Cancer Registries standards for determining cases of cancer, making use of multiple sources of information, including imaging reports, histology and pathology, cancer clinic visits and death certificates. ${ }^{15}$ Cancer is a reportable disease in Nova Scotia. The registry adheres to the standards set by the Canadian Cancer Registry, maintained at Statistics Canada, and by the North American Association of Central Cancer Registries, which is primarily SEER (surveillance, epidemiology and end results) based. These standards govern the collection and coding of all core data variables, including date of diagnosis. Quality and data integrity are maintained by checks performed at the time of data entry, and at annual submissions to national and international cancer reporting agencies.

\section{Registry date of diagnosis}

Date of diagnosis is determined according to the Canadian Council of Cancer Registries standard, which prioritizes sources in the following hierarchical manner: cytology, pathology, imaging, clinical and death certificate. For example, lung cancer that is first diagnosed through imaging but later confirmed through biopsy (cytology) will be registered with a diagnosis date consistent with the biopsy, not the imaging.

\section{Case demographics, stage and survival}

Data elements extracted from the registry included patient age at diagnosis, patient sex, histology, cancer stage at diagnosis and patient date of death. Data were extracted for all cases diagnosed in the 2014 calendar year, with death data complete through Aug. 31, 2016. Survival was calculated from the registry date of diagnosis to date of death. Registry data were extracted by database administrators at the Provincial Program for Cancer Care and not by the authors of this study.

\section{Route to diagnosis}

Diagnostic imaging for each case was reviewed to identify the first imaging report that suggested the possibility of lung cancer (A.S., D.M.). All radiographic and computed tomography (CT) reports in the province are available on a single Provincial Picture Archiving and Communication System used clinically. There is no private radiographic or CT imaging done outside of this network.
The requisition point of origin was identified by examining the requisition form associated with the first report raising the possibility of lung cancer (A.S., D.M.). In most cases, the requisition was also scanned into the same PACS. For a small number of cases, site visits were required to access paper-based charts for paper-only requisitions. If imaging was not the initial positive diagnostic test, pathology records were reviewed through electronic hospital records (A.S.). Cases were then assigned a route to diagnosis (emergency department or non-emergency department), based on the point of origin of the first requisition. This process is summarized in Figure 1.

\section{Primary care access}

The presence or absence of a family physician is charted as a standard of practice for every patient who presents to an emergency department in Nova Scotia. Nurse practitioners also provide primary care in Nova Scotia, but do so only in association with a physician. These data were obtained from electronic and paper-based emergency department charts (A.S.). Determining the presence of a family physician for the non-emergency department route was completed through electronic and paper hospital chart review of clinic visits (including oncology, thoracic surgery and palliative care) or death certificates (A.S.). Patients may not self-refer for diagnostic imaging, biopsy or specialist consultation in Nova Scotia. Patients who receive these services would have a referring physician but not necessarily a primary care provider. In 2014 there were no lung cancer-specific clinics in the province; the diagnostic process could be guided by primary care or by specialist. Referrals for work-up or treatment could be sent to internal medicine, respirology (pulmonology), oncology or thoracic surgery.

\section{Clinical indication for testing}

Test requisitions, dictated physician notes and written emergency physician notes were reviewed to establish the clinical indication for the testing that led to diagnosis (symptomatic, incidental, screening or surveillance) (A.S., D.M.). For many of the emergency departments, the triage note (presenting complaint) is used as the imaging requisition. For cases in which lung cancer was initially suspected based on a pathology or histology result, the dictated clinic notes were reviewed to determine the indication for testing. Cases were classified as symptomatic if the requisition included signs and symptoms of lung cancer as defined by the provincial guidelines for the diagnosis and referral of suspected lung cancer developed by Cancer Care Nova Scotia ${ }^{16}$ (Appendix 1, available at www.cmajopen.ca/content/7/1/E117/suppl/DC1). Incidental cases were defined as those for which the clinical indication for testing that prompted diagnosis met none of the aforementioned criteria (e.g., colon cancer staging, major trauma).

A separate designation of "surveillance" was assigned to cases for which an incidental finding of a small pulmonary nodule of low suspicion resulted in further surveillance imaging, only later showing suspicion for cancer. A small number 
of cases diagnosed as a result of a nationwide clinical trial (Pan-Canadian Early Detection of Lung Cancer Study) ${ }^{17}$ were indexed under the category of "screening." This category also included cases in patients who were not involved in clinical trials but for whom the imaging requisition specifically stated "screening."

\section{Quality assurance}

The details regarding the Canadian Cancer Registry, including data integrity and quality, have been described elsewhere. ${ }^{18}$ Data not collected through the registry were independently reviewed by 2 physicians (A.S. and a research assistant who was not an author of this study). Comparison of data extraction allowed for the identification of both data entry errors (route to diagnosis) and uncertainties regarding clinical indication for testing. All disagreements were settled by a third, more senior physician (D.M.). Formal interrelater reliability testing was not performed. Validation studies were not performed. The decision tree regarding the route to diagnosis and indication for testing was not externally validated.

\section{Data analysis}

Descriptive statistics are presented for the sample and stratified by route to diagnosis. Kaplan-Meier survival curves and associated logrank tests were used to compare route of presentation; in addition, Kaplan-Meier curves were stratified by stage at diagnosis. Cox regression modelling (hazard rate ratios [HRRs] and 95\% confidence intervals [CIs]) was used to compare survival as a function of route of diagnosis, controlling for common confounders (age, sex) and stage. Statistical analyses were carried out using SPSS (IBM SPSS, version 23).

\section{Ethics approval}

This project received approval from the Institutional Research Ethics Board of the Nova Scotia Health Authority.

\section{Results}

\section{Case identification}

We identified 972 cancers recorded in the registry in 2014, which corresponded to 951 unique patients. Data were incomplete for 5 patients: 3 patients underwent initial testing out of province, and 2 patients had no relevant imaging that indicated suspicion for lung cancer and no alternate method of diagnosis as per registry data. Thus, our analysis included data from 946 patients. Stage-specific analysis excluded cases of cancer for which staging was listed as not available (14 cases), occult ( 3 cases) or stage 0 (9 cases). One case each of lymphangioleiomyomatosis, myofibroblastic tumour and carcinoid tumourlet were excluded from stage-specific analysis.

\section{Route to diagnosis}

Diagnostic imaging (e.g., radiography or CT) was the initial positive test for all but 5 cases. Two cases were diagnosed through tissue and 3 were diagnosed via "death certificate only," with no positive testing before death. About one-third of cases $(336,35.5 \%)$ were diagnosed through the emergency department.

\section{Patient demographics, disease stage and survival}

Differences in demographics and stage at presentation between patients whose cancer was diagnosed through the emergency department and non-emergency department routes are summarized in Table 1 . Although there were no differences in terms of patient age by route to diagnosis, men were slightly more likely to receive their diagnosis through the emergency department $(37.1 \%, 182 / 491)$ than women $(33.8 \%, 154 / 455)$.

Cancer that was detected through the emergency department was more likely to be at an advanced stage, and patients were more likely to have a shorter survival than those whose

Table 1: Characteristics of patients with lung cancer in Nova Scotia by route to diagnosis, 2014

\begin{tabular}{|c|c|c|c|}
\hline \multirow[b]{2}{*}{ Characteristic } & \multicolumn{3}{|c|}{ No. $(\%)^{*}$} \\
\hline & $\begin{array}{l}\text { Overall } \\
n=946\end{array}$ & $\begin{array}{c}\text { Diagnosis } \\
\text { through ED } \\
n=336\end{array}$ & $\begin{array}{c}\text { Diagnosis } \\
\text { through non-ED } \\
\quad n=610\end{array}$ \\
\hline \multicolumn{4}{|l|}{ Sex } \\
\hline Male & $491(51.9)$ & $182(54.2)$ & $309(50.7)$ \\
\hline Female & $455(48.1)$ & $154(45.8)$ & 301 (49.3) \\
\hline $\begin{array}{l}\text { Age, yr, } \\
\text { mean } \pm \text { SD }\end{array}$ & $70.3 \pm 11$ & $71.2 \pm 11$ & $69.7 \pm 10$ \\
\hline Age range, yr & $18-98$ & $33-98$ & $18-97$ \\
\hline \multicolumn{4}{|l|}{ Age group, yr } \\
\hline$\leq 60$ & $163(17.2)$ & $56(16.7)$ & $107(17.5)$ \\
\hline $60-69$ & $265(28.0)$ & $84(25.0)$ & $181(29.7)$ \\
\hline $70-79$ & $348(36.8)$ & $129(38.4)$ & 219 (35.9) \\
\hline$\geq 80$ & $170(18.0)$ & 67 (19.9) & 103 (16.9) \\
\hline \multicolumn{4}{|c|}{ Indication for testing } \\
\hline Symptomatic & $751(79.4)$ & 314 (93.5) & 437 (71.6) \\
\hline Incidental & $78(8.2)$ & $12(3.6)$ & $66(10.8)$ \\
\hline Surveillance & $104(11.0)$ & $10(3.0)$ & $94(15.4)$ \\
\hline Screening & $13(1.4)$ & $0(0.0)$ & $13(2.1)$ \\
\hline \multicolumn{4}{|l|}{ Stage at diagnosis } \\
\hline Unknown & $14(1.5)$ & $4(1.2)$ & $10(1.6)$ \\
\hline Occult & $3(0.3)$ & $3(0.9)$ & $0(0.0)$ \\
\hline 0 & $9(1.0)$ & $1(0.3)$ & $8(1.3)$ \\
\hline I & $218(23.0)$ & $49(14.6)$ & $169(27.7)$ \\
\hline II & $82(8.7)$ & $28(8.3)$ & $54(8.9)$ \\
\hline III & $155(16.4)$ & $51(15.2)$ & $104(17.0)$ \\
\hline IV & 465 (49.2) & $200(59.5)$ & $265(43.4)$ \\
\hline $\begin{array}{l}\text { No. of deaths (to } \\
\text { Aug. } 31,2016 \text { ) }\end{array}$ & $592(62.5)$ & $247(73.1)$ & $345(56.7)$ \\
\hline
\end{tabular}




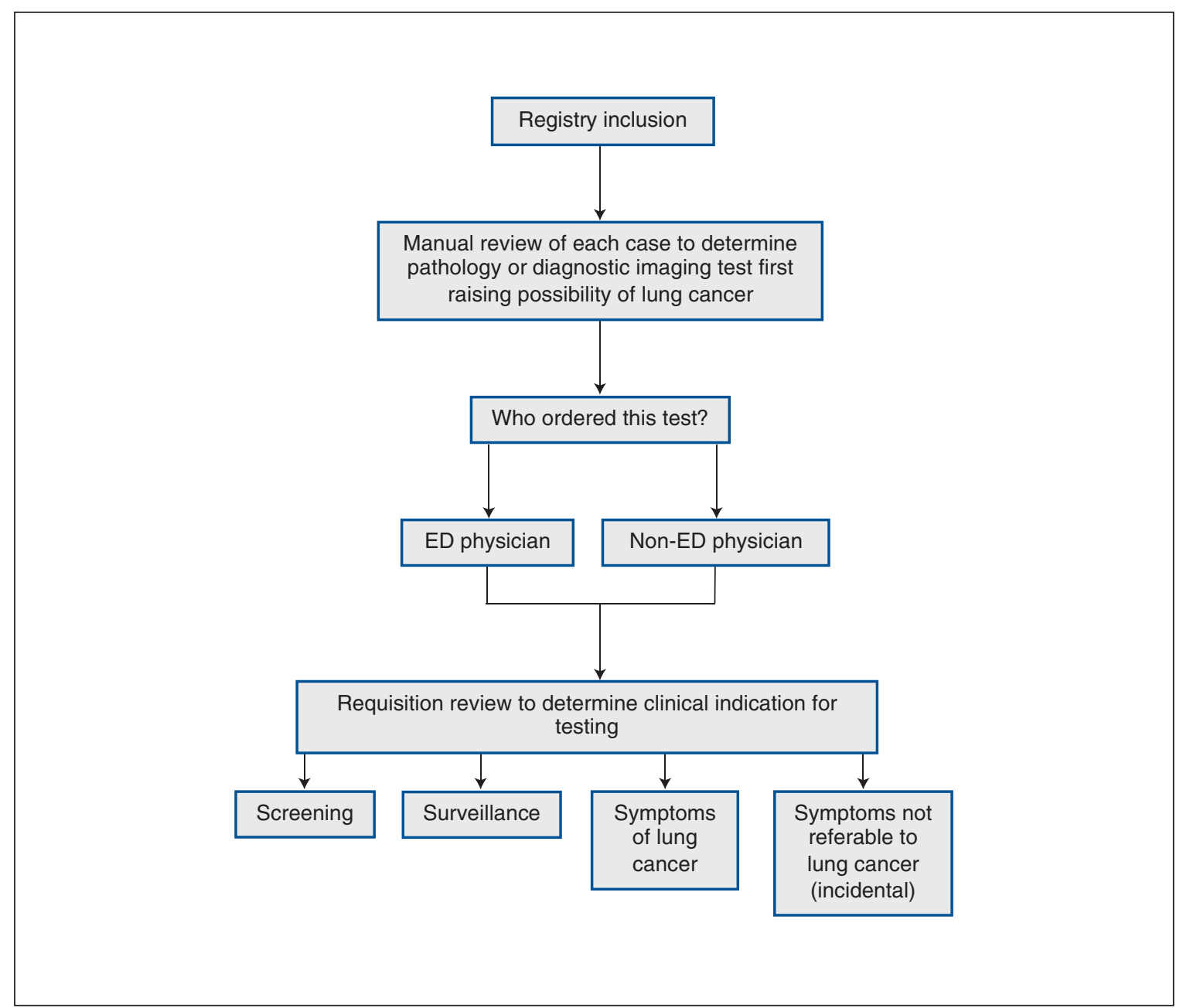

Figure 1: Case review process to determine route to diagnosis. ED = emergency department.

cancer was diagnosed outside the emergency department (Figure 2). One-year survival from registry date of diagnosis was $28.4 \%$ in the emergency department route to diagnosis and $49.5 \%$ in the non-emergency department group. The difference in survival by route of diagnosis was evident for each stage of disease and was significantly different at stages III and IV (Figure 3).

Cox regression analysis showed an increased risk of death associated with presentation via the emergency department (HRR 1.87, 95\% CI 1.60-2.18). This relationship held after adjustment for age and stage as confounders (HRR 1.54, 95\% CI 1.32-1.81). There was no confounding by sex (i.e., no change in HRR).

\section{Primary care access}

Information regarding the presence of a family physician was available for all patients whose cancer was diagnosed through the emergency department (336), but only for $77.2 \%$ of the patients whose cancer was diagnosed outside of the emergency department route (471). The lack of a primary care provider was documented for $24(7.1 \%)$ patients who presented through the emergency department and $8(1.7 \%)$ who presented elsewhere. Patients in the non-emergency department group who did not have a primary care provider accounted for $1.1 \%$ of cases in the early stage (stage I or II) and $2.2 \%$ of cases in the late stage (III or IV). For the emergency department group, $2.6 \%$ of cases diagnosed at early stage and $8.0 \%$ of the late stage cases were in patients who reported having no family physician.

\section{Clinical indication for testing}

In both the emergency and non-emergency department routes, most (overall 751, 79.4\%) cancers were initially detected through investigation of signs or symptoms associated with lung cancer. Further data are included in Table 1.

\section{Quality assurance}

Twelve patients were reassigned between emergency department and non-emergency department routes to diagnosis after comparison of data entry ( 7 from emergency department to non-emergency department; 5 from non-emergency department to emergency department) owing to data entry error. The senior author (D.M.) reviewed 14 files regarding clinical indication owing to uncertainty or disagreement. 


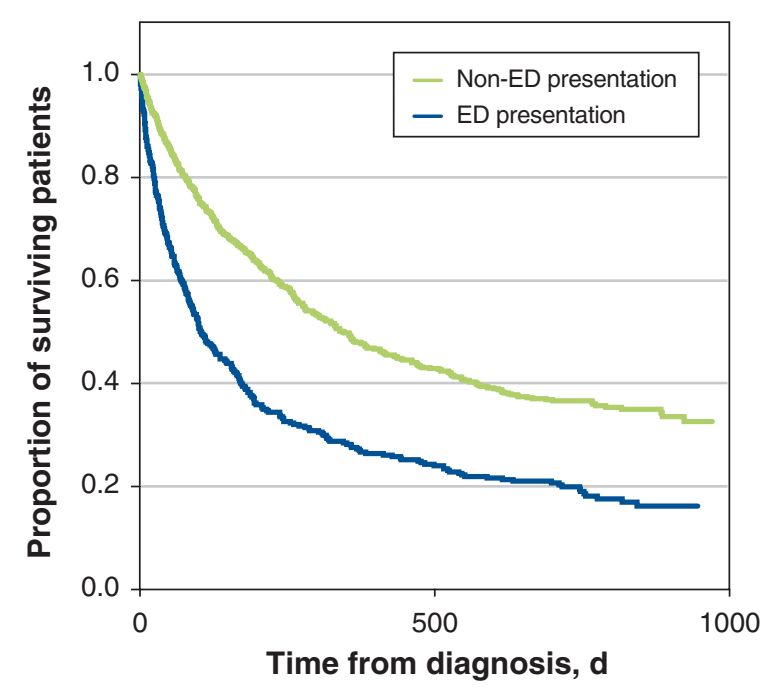

Figure 2: Survival of patients in Nova Scotia with lung cancer by route to diagnosis. ED = emergency department.

\section{Interpretation}

More than one-third of lung cancer diagnoses in Nova Scotia in 2014 were initiated through a visit to the emergency department. We found substantial differences in stage at diagnosis by route of diagnosis, with a disproportionate representation of advanced cancers in the emergency department group, and a resulting shorter survival for patients in this group.

Although there is a lack of published comparative Canadian data, our results are similar to those of a study done in the United Kingdom that showed that 39\% of lung cancer diagnoses were settled after an emergency consultation. ${ }^{19} \mathrm{~A}$ 2009 New Zealand study found that $36 \%$ of patients with lung cancer initially presented through the emergency department. ${ }^{4}$ This consistency suggests that our findings are likely transferable to other jurisdictions with similar health care settings. Lower numbers (13\% and 19\%) have been found in other studies done in $\mathrm{Japan}^{20}$ and the UK, ${ }^{21}$ respectively, but these studies more narrowly defined emergency department presentation by including only patients who were admitted to hospital from the emergency department. Additional research from the UK has found lower survival for patients who present through the emergency department. ${ }^{22,23}$

Barriers in access to primary care have been suggested to drive a higher proportion of nonemergent cases to the emergency department. ${ }^{4}$ Although there was a higher percentage of "orphaned" patients in the emergency department route to diagnosis, compared with the non-emergency department route, the percentage in both groups was relatively small. For comparison, data from Statistics Canada for 2014 shows that $10.6 \%$ of Nova Scotians reported not having a regular health care provider. ${ }^{24}$ More in-depth research has shown that patients who initially present to the emergency department and are later found to have lung cancer are often selfreferred; socioeconomic deprivation and potential financial, geographic, cultural or information barriers are postulated as contributing factors. ${ }^{4,22}$ The nonspecific nature of lung cancer symptoms, in addition to psychosocial factors such as patient guilt associated with smoking ${ }^{6-9,22,25}$ have also been suggested as reasons for delays in seeking care. In turn, these nonspecific symptoms may be less likely to prompt expedient assessment and referral by family physicians. In our study, only 38 patients $(4.0 \%$ of total) presented with hemoptysis, an alarming sign that usually generates urgent medical work-up.

A multidisciplinary subspecialist approach has been suggested as a requirement for optimal patient treatment. ${ }^{26,27}$ Already extremely limited in Canada outside of urban centres, access to experts (including subspecialty thoracic radiology, oncology and surgery) is further restricted when required "after hours" in an emergency setting. In addition, the late presentation of disease to the emergency department may present a greater strain on already stretched resources and, by extension, on diagnostic and consulting resources with many patients presenting after 7:00 pm. ${ }^{28}$

\section{Limitations}

We captured all cases of lung cancer in the province via a registry that follows Canadian Council of Cancer Registries standards for data collection. However, no validation studies were performed to review the additional data not collected through the registry.

Owing to the single province-wide imaging archive system, we were able to retrieve all relevant imaging data. Although precipitous emergency department presentation may suggest diagnostic delays (i.e., wait times for CT and specialist appointments), there is currently no wait time for general radiography in the province. Provided work-up was initiated with radiography, concerning imaging reports would have been accurately identified and linked to the appropriate route of entry in our study. Cases diagnosed through the emergency department were therefore unlikely to be waiting for imaging or specialist referral by a family doctor. However, there may have been incomplete capture of patients waiting for specialist referral after a normal radiograph but before CT, because primary care records were inaccessible. The approach to assigning route of diagnosis was not validated. However, the review approach was unlikely to have biased the results given that it was done by 2 physicians independently. In addition, although our results suggest that nearly $80 \%$ of lung cancer presents symptomatically, this is likely an overestimate. The ability to distinguish symptomatic lung cancer diagnosis from incidental diagnosis was limited owing to the nonspecific nature of symptoms. Many instances were noted where lung cancer was unlikely to have been the cause of the respiratory symptoms being investigated. There was no objective way to ensure that lung cancer was not responsible for at least a component of 


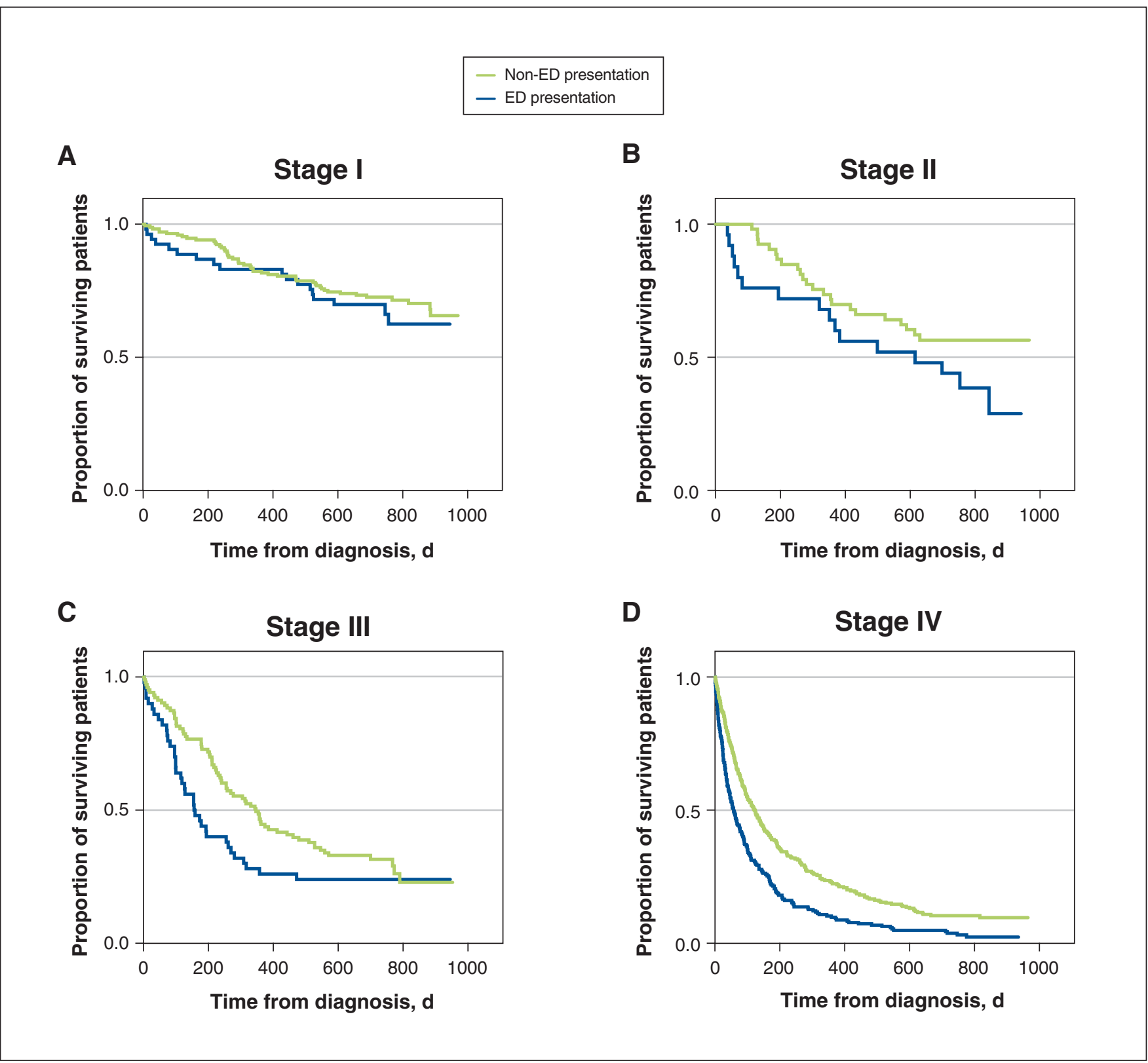

Figure 3: Comparison of survival of patients in Nova Scotia with lung cancer by route to diagnosis, parsed by stage at diagnosis. ED = emergency department.

the symptoms, even in a patient with a competing diagnosis. In addition, data regarding the presence of a primary care provider for patients in the non-emergency department route to diagnosis were unavailable in almost one-quarter of cases.

\section{Conclusion}

Strategies to promote early recognition, to improve resources for patient navigation after visiting the emergency department and to prevent unnecessary delays along the diagnostic pathway may all be important to improving survival for patients with lung cancer. Despite current efforts to target family physicians and lung specialists, the consistent presentation of lung cancer in our acute care facilities reinforces the need for consensus guidelines, resources and training tailored specifically for the emergency department.

\section{References}

1. Cancer [fact sheet]. Geneva: World Health Organization; 2018. Available: www.who.int/news-room/fact-sheets/detail/cancer (accessed 2018 Aug. 30).

2. Canadian Cancer Statistics Advisory Committee. Canadian cancer statistics 2018. Toronto: Canadian Cancer Society; 2018. Available: www.cancer.ca/Canadian -Cancer-Statistics-2018-EN (accessed 2018 Aug. 30).

3. Walters S, Maringe C, Coleman MP, et al.; ICBP Module 1 Working Group. Lung cancer survival and stage at diagnosis in Australia, Canada, Denmark, Norway, Sweden and the UK: a population-based study 2004-2007. Thorax 2013;68:551-64.

4. Beatty S, Stevens W, Stevens G, et al. Lung cancer in patients in New Zealand initially present to secondary care through the emergency department rather than by referral to a respiratory specialist. N Z Med F 2009;122:33-41. 
5. Corner J, Hopkinson J, Fitzsimmons D, et al. Is late diagnosis of lung cancer inevitable? Interview study of patients' recollections of symptoms before diagnosis. Thorax 2005;60:314-9.

6. Dunn J, Garvey G, Valery PC, et al. Barriers to lung cancer care: health professionals' perspectives. Support Care Cancer 2017;25:497-504.

7. Chapple A, Ziebland S, McPherson A. Stigma, shame, and blame experienced by patients with lung cancer: qualitative study. BM7 2004;328:1470.

8. Lehto RH. Patient views on smoking, lung cancer, and stigma: a focus group perspective. Eur 7 Oncol Nurs 2014;18:316-22.

9. LoConte NK, Else-Quest NM, Eickhoff J, et al. Assessment of guilt and shame in patients with non-small-cell lung cancer compared with patients with breast and prostate cancer. Clin Lung Cancer 2008;9:171-8.

10. Cancer Care Manitoba referral guidelines: Timeline model in Manitoba for the lung cancer patient journey from suspicion of cancer to treatment in sixty days [1 screen]. Winnipeg: CancerCare Manitoba. Available: www.cancercare. $\mathrm{mb}$.ca/export/sites/default/For-Health-Professionals/.galleries/files/diagnostic -pathway-files/lung-diagnostic-pathway-files/IN60_LC_FullPath_19-09-2014. pdf (accessed 2017 July 28).

11. Del Giudice L, Young S, Vella E, et al. Referral of suspected lung cancer by primary care practitioners. Toronto: Cancer Care Ontario; 2011. Available: www.cancercareontario.ca/en/guidelines-advice/types-of-cancer/216 (accessed 2018 Nov. 26).

12. Melling PP, Hatfield AC, Muers MF, et al. Lung cancer referral patterns in the former Yorkshire region of the UK. Br 7 Cancer 2002;86:36-42.

13. Gorham J, Ameye L, Berghmans T, et al. The lung cancer patient at the emergency department: a three-year retrospective study. Lung Cancer 2013;80: 203-8.

14. Need A Family Practice data. Halifax: Nova Scotia Health Authority. Available: www.nshealth.ca/need-family-practice-data (accessed 2018 Aug. 30).

15. Canadian Cancer Registry (CCR): data sources and methodology. Ottawa: Statistics Canada; modified 2018 Jan. 26. Available: http://www23.statcan.gc.ca/ $\mathrm{imdb} / \mathrm{p} 2 \mathrm{SV}$.pl? Function=getSurvey \&SDDS=3207\&lang=en $\& \mathrm{db}=\mathrm{imdb} \& \mathrm{adm}=$ $8 \&$ dis $=2$ (accessed 2018 Sept. 26).

16. Suspected lung cancer - Pathway to a timely diagnosis: guidelines for the diagnosis and referral of suspected lung cancer. Halifax: Nova Scotia Health Authority; 2016.

17. Tammemagi MC, Schmidt H, Martel S, et al. Participant selection for lung cancer screening by risk modelling (the Pan-Canadian Early Detection of Lung Cancer [PanCan] study): a single-arm, prospective study. Lancet Oncol 2017;11:1523-31.

18. Canadian Cancer Registry (CCR): data accuracy. Ottawa: Statistics Canada; modified 2018 Jan. 26. Available: http://www23.statcan.gc.ca/imdb/p2SV.pl? Function=getSurvey\&SDDS=3207\#a3 (accessed 2018 Sept. 26).

19. Elliss-Brookes L, McPhail S, Ives A, et al. Routes to diagnosis for cancer determining the patient journey using multiple routine data sets. $\mathrm{Br} F$ Cancer 2012;107:1220-6.

20. Fujimoto D, Shimizu R, Morimoto T, et al. Analysis of advanced lung cancer patients diagnosed following emergency admission. Eur Respir 7 2015;45: 1098-107.
21. Beckett P, Tata LJ, Hubbard RB. Risk factors and survival outcome for nonelective referral in non-small cell lung cancer patients - analysis based on the National Lung Cancer Audit. Lung Cancer 2014;83:396-400.

22. Tataru D, Jack RH, Lind MJ, et al. The effect of emergency presentation on surgery and survival in lung cancer patients in England, 2006-2008. Cancer Epidemiol 2015;39:612-6.

23. McPhail S, Elliss-Brookes L, Shelton J, et al. Emergency presentation of cancer and short-term mortality. Br 7 Cancer 2013;109:2027-34.

24. Access to a regular medical doctor, 2014. Ottawa: Statistics Canada; modified 2015 Nov. 27. Available: https://www150.statcan.gc.ca/n1/pub/82-625 -x/2015001/article/14177-eng.htm (accessed 2018 Aug. 30).

25. Friedemann Smith C, Whitaker KL, Winstanley K, et al. Smokers are less likely than non-smokers to seek help for a lung cancer 'alarm' symptom. Thorax 2016;71:659-61.

26. Sculier JP, Vansteenkiste J, Schönfeld N, et al. Thoracic oncology in Europe: the ERS action plan by the Thoracic Oncology Assembly. Eur Respir 7 2010;36:1227-8.

27. Gamarra F, Boffetta P, De Ruysscher D, et al. Thoracic Oncology HERMES syllabus: setting the basis for thoracic oncology training in Europe. Eur Respir 7 2013;42:568-71.

28. Minami S, Yamamoto S, Ogata Y, et al. Emergency department visits after hours by lung cancer patients in Japan. Support Care Cancer 2013;21:2443-51.

Affiliations: Departments of Diagnostic Radiology (Suhail, Crocker, Payne, Manos), Psychiatry (Crocker) and Emergency Medicine (Das), Dalhousie University, Halifax, NS

Contributors: All of the authors contributed to the design of the study and to the analysis and interpretation of the data. All of the authors were involved in drafting and revising the manuscript and gave final approval of the version to be published. All of the authors agreed to be accountable for all aspects of the work.

Funding: This project was funded by a grant from the Dalhousie Radiology Research Foundation.

Acknowledgements: The authors thank the Nova Scotia Health Authority and the Nova Scotia Department of Health and Wellness for registry access. Any opinions expressed in this paper do not necessarily reflect the views of these organizations. The authors also thank Maureen MacIntyre and Devbani Raha for assistance with registry interpretation, and Dr. Victoria Luong for her expert assistance with hospital record review.

Supplemental information: For reviewer comments and the original submission of this manuscript, please see www.cmajopen.ca/content/7/1/ E117/suppl/DC1. 